Article

\title{
Next-generation matrices and basic reproductive numbers for all phases of the Coronavirus disease
}

\author{
Gabriel Obed Fosu' ${ }^{1, *}$, Emmanuel Akweittey ${ }^{1}$ and Albert Adu-Sackey ${ }^{2}$ \\ 1 Department of Mathematics, Presbyterian University College, Ghana. \\ 2 Department of Applied Mathematics, Koforidua Technical University, Ghana. \\ * Correspondence: gabriel.obed@presbyuniversity.edu.gh
}

Received: 12 May 2020; Accepted: 1 June 2020; Published: 27 August 2020.

\begin{abstract}
During the early phase of Covid-19, the transmissibility of the coronavirus disease was estimated using the classical SIR and SEIR models. However, with the advent of some controlling measures in its informative stages, these classical compartmental models have been ameliorated to provide accurate insight of the coronavirus disease. The paper seeks to derive the basic reproductive formulas for these improved models using the matrix approach. These transmissibility equations detail the dynamics of the coronavirus disease for all phases of the pandemic; either the infected population is on lockdown or not; either infectious persons are quarantined or not; either a vaccination program has been rolled out or yet to be rolled out. With the availability of data, any of these transmissibility equations could be adopted to report on the endemicity of the coronavirus disease.
\end{abstract}

Keywords: Basic reproductive number, Covid-19, compartmental epidemic models, next-generation matrix.

MSC: 62D05.

\section{Introduction}

I he paper presents the basic reproductive formulas for several enhanced coronavirus models. These formulae highlight the transmissibility of the coronavirus disease regardless of its states. The basic reproductive equation and its corresponding number is a measure of the transmissibility of a viral disease. It is often denoted by $R_{0}$ (R nought). It measures the mean number of new infections assuming an infectious person is introduced into a susceptible or naive population and is one of the fundamental concepts in infectious disease modeling. The basic reproductive number gives a simple and clear explanation of the growth or decay of an endemic disease. With a threshold value of unity, it is theorized that a disease will invade a susceptible population when $R_{0}$ is greater than this threshold value. On the other hand, the disease will die-out when $R_{0}$ is less than one [1,2]. Related to the basic reproductive number is the Malthusian or exponential growth parameter $R$ [3]. With the Malthusian parameter classification, the disease will invade the host population when $R$ is greater than zero, but dies-off when $R$ is less than zero.

Generally, the basic reproductive formulas are derived using three main approaches, that is statistical[4,5], stochastic [6,7], and mathematical [8-10]. The mathematics has several subdivisions and the most common is the use of the matrix (NGM). In all cases, the basic reproductive formula is obtained from the Jacobian matrix. The Jacobian matrix is the first partial derivatives of the given system of equations. The differential equations are also deduced from the compartmental diagrams.

During the early phase of the corona pandemic, [11], used the $R_{0}$ from an SIR model to explain the transmissibility of SARS-CoV-2 for some selected cities in Italy. The authors estimated that the basic reproductive number for these nine cities to range between 2.43 and 3.10. In a similar research by [12], the $R_{0}$ for Iran was also inferred from an SIR model. The value was estimated to lie within the neighborhood of 4.8 during the first week of the coronavirus pandemic but reduced drastically to a value less than one after the seventh week. In Wuhan where the virus erupted, the basic reproductive number was estimated in the range between 2.8 and 3.9 based an SEIR model [13]. The paper stated that the transmissibility of SARS-CoV-2 during the early stages of infection was higher than SARS-CoV.

Though the $R_{0}$ of the coronavirus disease has been addressed in several papers, these values may not replicate the transmissibility of the virus within its informative stages. These exiting values were 
estimated during the earlier phase of Covid-19, where there were inaccurate data on the spread and control of SARS-CoV-2. In the recent past, new compartmental corona models have been developed to appropriately explain the dynamic of the coronavirus disease depending on the eradication strategies adopted to control the spread in a naive population [14]. It is therefore imperative to derive the corresponding $R_{0}$ equations to properly reflect the transmissibility of the virus. The basic reproductive formulas are derived using the next-generation matrix approach. Here, the NGM with three different domains is considered in deriving the transmissibility equations. [2] presents a detailed explanation of these domain classifications.

The mathematics of the next-generation matrix is presented in Section 2. In Section 3, the $R_{0}$ equations for eight Covid-19 models are presented. For each model, three different NGM domains are employed to derive the $R_{0}$ equations. Section 4 provides a general summary of the entire work.

\section{Next-generation matrices}

This method was first introduced by [15] but was further elaborated by [16]. To derive the basic reproductive number $\left(R_{0}\right)$ using the next-generation matrix, the Jacobian matrix $(J)$ for the infected sub-population is decomposed into a sum of two matrices; the transmission matrix $(\Gamma)$ and the transition matrix $(\Delta) . \Gamma$ accounts for the number of new infections, while $\Delta$ used to characterize movement to-and-fro compartments. The transmission and transition matrices are used during the computation process of the NGM. Besides, the $R_{0}$ equations are the dominant eigenvalues or spectral radius of the NGM.

Here, the NGM is categorized under three domain headings; a large domain $\left(D_{L}\right)$, classical domain $\left(D_{C}\right)$, and small domain $\left(D_{S}\right)$.

The spectral formula for the NGM with large domain is given by

$$
D_{L}=-\Gamma \Delta^{-1}
$$

Similarly, the NGM with the classical domain is deduced from Equation (1) as

$$
D_{C}=\Psi^{\prime} D_{L} \Psi=-\Psi^{\prime} \Gamma \Delta^{-1} \Psi
$$

where $\Psi$ is an arbitrary matrix composed of unit column vectors $\left(e_{i}\right)$ such that the $i$ th row of the transmission matrix is not zero [2].

The dimension of the large domain could be reduced to obtain the result of the NGM with small domain. For the small domain, the transmission matrix $(\Gamma)$ is dichotomized into two separate vectors $(\Omega, \Lambda)$. A row vector $(\Omega)$, and a column vector $(\Lambda)$ are defined to satisfy Equation (3).

$$
\Gamma_{i j}=\Lambda_{i} \Omega_{j}
$$

Then the NGM with the small domain is defined as

$$
D_{S}=-\Omega \Delta^{-1} \Lambda
$$

For the small domain, the spectral radius of this matrix (4) is also the basic reproductive formula. With the other two domains, the trace of the spectral formula is the basic reproductive equation. These domain analyzes are summarized by the following propositions.

Proposition 1. The NGM with classical domain $D_{C}$ and the NGM with large domain $D_{L}$ have the same non-zero eigenvalue.

Proof. Let $\Theta$ be an eigenvector of $D_{C}$ with corresponding eigenvalues $Y$. Then $D_{C} \Theta=-\Psi^{\prime} \Gamma \Delta^{-1} \Psi \Theta=Y \Theta$. Multiply this identity by $\Psi$ to get $-\Psi \Psi^{\prime} \Gamma \Delta^{-1} \Psi \Theta=Y \Psi \Theta$. But $\Psi \Psi^{\prime} \Gamma=\Gamma$, so $\Psi \Theta$ is an eigenvector of $D_{L}$ with corresponding eigenvalue $Y$, and the non-zero eigenvalues of $D_{C}$ and $D_{L}$ are the same.

Proposition 2. The NGM with small domain $D_{S}$ and the NGM with large domain $D_{L}$ have the same non-zero eigenvalue. 
Proof. Let $\Theta$ be an eigenvector of $D_{S}$ with corresponding eigenvalues $Y$. Then $D_{S} \Theta=-\Omega \Delta^{-1} \Lambda \Theta=Y \Theta$. Multiply this identity by $\Lambda$ to get $-\Lambda \Omega \Delta^{-1} \Lambda \Theta=\mathrm{Y} \Lambda \Theta$. But $\Lambda \Omega=\Gamma$. So $\Lambda \Theta$ is an eigenvector of $D_{L}$ with corresponding eigenvalue $\mathrm{Y}$.

Remark 1. The NGM with domains $D_{L}, D_{C}$ and $D_{S}$ have the same rank, same non-zero spectrum and same dominant eigenvalue.

\section{Covid-19 models and their basic reproductive equations}

The compartmental models discussed in this paper are detailed in [14]. The following notations are used to represent the various sub-populations: $S \rightarrow$ susceptible, $I \rightarrow$ infected, $E \rightarrow$ exposed, $R \rightarrow$ removed or recovered, $L \rightarrow$ lockdown, $Q \rightarrow$ quarantine, $V \rightarrow$ vaccinated, $I_{a} \rightarrow$ asymptomatic patients, and $I_{s} \rightarrow$ symptomatic patients. The parametric constants used for the formulation of these models are summarized in Table 1 . We begin by considering the simple SEIR model.

Table 1. Model Parameters and Their Definitions

\begin{tabular}{||cc||}
\hline Parameters & Definition \\
$\gamma \mu$ & Birth rate \\
$\alpha_{3}$ & Recovery rate $I_{a}$ \\
$\alpha_{4}$ & Recovery rate of $I_{S}$ \\
$\epsilon$ & Incubation/latency rate \\
$\alpha_{1}$ & Outflow from $I$ to $R$ \\
$\alpha_{2}$ & Outflow from $Q$ to $R$ \\
$\beta$ & Outflow from $S$ to $I$ \\
$\beta_{1}$ & Outflow from $S$ to $E$ \\
$\xi$ & Outflow from $E$ to $S$ \\
$\kappa$ & Outflow from $E$ to $I_{S}$ \\
$\phi$ & Outflow from $I_{a}$ to $I_{S}$ \\
$\theta_{1}$ & Outflow from $I$ to $Q$ \\
$\theta_{2}$ & Outflow from $E$ to $Q$ \\
$\eta$ & Outflow from $S$ to $L$ \\
$\delta$ & Outflow from $L$ to $S$ \\
$\omega_{1}$ & Outflow from $S$ to $V$ \\
$\tau$ & Infectivity factor \\
\hline
\end{tabular}

\subsection{SEIR Model}

The simple SEIR model that characterizes the coronavirus is given by the system (5).

$$
\left\{\begin{array}{l}
S^{\prime}(t)=\mu N-\beta_{1} S I-\gamma S+\xi E \\
E^{\prime}(t)=\beta_{1} S I-(\gamma+\epsilon+\xi) E \\
I^{\prime}(t)=\epsilon E-\left(\gamma+\alpha_{1}\right) I \\
R^{\prime}(t)=\alpha_{1} I-\gamma R .
\end{array}\right.
$$

The infectious subsystem is deduced from Equation (5) as

$$
\left\{\begin{array}{l}
E^{\prime}(t)=\beta_{1} S I-(\gamma+\epsilon+\xi) E ; \\
I^{\prime}(t)=\epsilon E-\left(\gamma+\alpha_{1}\right) I .
\end{array}\right.
$$

Then the Jacobian matrix from the system (6) is computed as

$$
J_{(E, I)}=\left[\begin{array}{cc}
-(\gamma+\epsilon+\xi) & \beta_{1} S \\
\epsilon & -\left(\gamma+\alpha_{1}\right)
\end{array}\right] .
$$


The Jacobian matrix is decomposed into two matrices as shown below.

$$
\Gamma=\left[\begin{array}{cc}
0 & \beta_{1} S \\
0 & 0
\end{array}\right], \quad \Delta=\left[\begin{array}{cc}
-(\gamma+\epsilon+\xi) & 0 \\
\epsilon & -\left(\gamma+\alpha_{1}\right)
\end{array}\right]
$$

Then the inverse of the transition matrix is also computed as

$$
\Delta^{-1}=\left[\begin{array}{cc}
\frac{-1}{\gamma+\epsilon+\xi} & 0 \\
\frac{-\epsilon}{\left(\gamma+\alpha_{1}\right)(\gamma+\epsilon+\xi)} & \frac{-1}{\gamma+\alpha_{1}}
\end{array}\right]
$$

The basic reproductive equation $\left(R_{0}\right)$ is derived by considering the following domain cases.

\section{Case I: NGM with large domain}

The NGM with large domain is denoted by $\left(D_{L}\right)$, that is

$$
D_{L}=-\Gamma \Delta^{-1}=\left[\begin{array}{cc}
\frac{\epsilon \beta_{1} S}{\left(\gamma+\alpha_{1}\right)(\gamma+\epsilon+\xi)} & \frac{\beta_{1} S}{\gamma+\alpha_{1}} \\
0 & 0
\end{array}\right]
$$

Therefore the basic reproductive formula considering a large domain matrix is

$$
R_{0}^{L}=\operatorname{trace}\left(D_{L}\right)=\frac{\epsilon \beta_{1} S}{\left(\gamma+\alpha_{1}\right)(\gamma+\epsilon+\xi)}
$$

\section{Case II: NGM with classical domain}

The NGM with classical domain is denoted by $D_{C}$. Here, we assume an auxiliary matrix $\Psi$ of the form: $\Psi=\left[\begin{array}{ll}1 & 0 \\ 0 & 0\end{array}\right]$. This implies that

$$
\Psi^{\prime} \Gamma=\left[\begin{array}{cc}
0 & \beta_{1} S \\
0 & 0
\end{array}\right] \text { and }-\Delta^{-1} \Psi=\left[\begin{array}{cc}
\frac{1}{\gamma+\epsilon+\xi} & 0 \\
\frac{\epsilon}{\left(\gamma+\alpha_{1}\right)(\gamma+\epsilon+\xi)} & 0
\end{array}\right]
$$

Then the spectral formula is given by

$$
D_{C}=-\Psi^{\prime} \Gamma \Delta^{-1} \Psi=\left[\begin{array}{cc}
\frac{\epsilon \beta_{1} S}{\left(\gamma+\alpha_{1}\right)(\gamma+\epsilon+\xi)} & 0 \\
0 & 0
\end{array}\right]
$$

Deductively, the basic reproductive number considering a classical domain matrix is given by the equation

$$
R_{0}^{C}=\operatorname{trace}\left(D_{C}\right)=\frac{\epsilon \beta_{1} S}{\left(\gamma+\alpha_{1}\right)(\gamma+\epsilon+\xi)}
$$

\section{Case III: NGM with small domain}

The NGM with small domain is denoted by $D_{S}$. We define an arbitrary row vector $\Omega$ and column vector $\Lambda$ such that $\Omega=\left[\begin{array}{ll}0 & \beta_{1}\end{array}\right]$ and $\Lambda=\left[\begin{array}{ll}S & 0\end{array}\right]^{\prime}$. Note that $\Gamma_{i j}=\Lambda_{i} \times \Omega_{j}$. This implies that

$$
-\Omega \Delta^{-1}=\left[\begin{array}{cc}
\frac{\epsilon \beta_{1}}{\left(\gamma+\alpha_{1}\right)(\gamma+\epsilon+\xi)} & \frac{\beta_{1}}{\gamma+\alpha_{1}}
\end{array}\right] .
$$

Then the basic reproductive formula for a small domain matrix is

$$
R_{0}^{S}=D_{S}=-\Omega \Delta^{-1} \Lambda=\frac{\epsilon \beta_{1} S}{\left(\gamma+\alpha_{1}\right)(\gamma+\epsilon+\xi)} .
$$


It could be realized that the $R_{0}$ result for these different domains are equivalent to each other. The susceptible population $S$ is assumed to equal unity for a naive population. Again, if we assume that birth rate $\mu$ offset death rate $\gamma$ then the basic reproductive number for the SEIR model reduce to

$$
R_{0}=\frac{\epsilon \beta_{1}}{\alpha_{1}(\epsilon+\xi)}
$$

Moreover, the $R_{0}$ value for the SEIR model reverts to SIR model when $\xi=0$. That is

$$
R_{0}=\frac{\beta_{1}}{\alpha_{1}}
$$

\section{2. $S E I_{S} I_{a} R$ Model}

The system of equations for an SEIR model with asymptomatic $\left(I_{a}\right)$ and symptomatic $\left(I_{s}\right)$ compartments is given by (8). This system is hereafter referred to as the $S E I_{s} I_{a} R$ model.

$$
\left\{\begin{array}{l}
S^{\prime}(t)=\mu N-\beta_{1} S\left(I_{s}+\tau I_{a}\right)-\gamma S+\xi E \\
E^{\prime}(t)=\beta_{1} S\left(I_{s}+\tau I_{a}\right)-(\gamma+\epsilon+\xi) E \\
I_{s}^{\prime}(t)=\epsilon k E+\phi I_{a}-\left(\gamma+\alpha_{4}\right) I_{s} \\
I_{a}^{\prime}(t)=\epsilon(1-k) E-\left[\gamma+\alpha_{3}+\phi\right] I_{a} \\
R^{\prime}(t)=\alpha_{4} I_{s}+\alpha_{3} I_{a}-\gamma R /
\end{array}\right.
$$

Equation (8) is reduced to form the infectious subsystem as (9).

$$
\left\{\begin{array}{l}
E^{\prime}(t)=\beta_{1} S\left(I_{s}+\tau I_{a}\right)-(\gamma+\epsilon+\xi) E \\
I_{s}^{\prime}(t)=\epsilon k E+\phi I_{a}-\left(\gamma+\alpha_{4}\right) I_{s} \\
I_{a}^{\prime}(t)=\epsilon(1-k) E-\left[\gamma+\alpha_{3}+\phi\right] I_{a}
\end{array}\right.
$$

The Jacobian matrix is deduced as

$$
J\left(E, I_{s}, I_{a}\right)=\left[\begin{array}{ccc}
-(\gamma+\epsilon+\xi) & \beta_{1} S & \tau \beta_{1} S \\
\epsilon k & -\left(\gamma+\alpha_{1}\right) & \phi \\
\epsilon(1-k) & 0 & -\left(\gamma+\alpha_{3}+\phi\right)
\end{array}\right]
$$

Then

$$
\Gamma=\left[\begin{array}{ccc}
0 & \beta_{1} S & 0 \\
0 & 0 & 0 \\
0 & 0 & 0
\end{array}\right], \Delta=\left[\begin{array}{ccc}
-(\gamma+\epsilon+\xi) & 0 & 0 \\
\epsilon k & -\left(\gamma+\alpha_{4}\right) & \phi \\
\epsilon(1-k) & 0 & -\left(\gamma+\alpha_{3}+\phi\right)
\end{array}\right]
$$

and

$$
\Delta^{-1}=\left[\begin{array}{ccc}
\frac{-1}{\gamma+\epsilon+\xi} & 0 & 0 \\
\frac{-\epsilon k\left(\gamma+\alpha_{3}+\phi\right)+\phi \epsilon(1-k)}{(\gamma+\epsilon+\xi)\left(\gamma+\alpha_{3}+\phi\right)} & \frac{-1}{\gamma+\alpha_{4}} & \frac{-\phi}{\left(\gamma+\alpha_{4}\right)\left(\gamma+\alpha_{3}+\phi\right)} \\
\frac{-\epsilon(1-k)}{(\gamma+\epsilon+\xi)\left(\gamma+\alpha_{3}+\phi\right)} & 0 & \frac{-1}{\gamma+\alpha_{3}+\phi}
\end{array}\right] .
$$

Similarly, the basic reproductive equation $\left(R_{0}\right)$ for this model is derived by considering the following domain cases.

\section{Case I: NGM with large domain}

$$
D_{L}=\left[\begin{array}{ccc}
\frac{\beta_{1} S[\epsilon k]\left(\gamma+\alpha_{3}+\phi\right)+\phi \epsilon(1-k)}{(\gamma+\epsilon+\xi)\left(\gamma+\alpha_{4}\right)\left(\gamma+\alpha_{3}+\phi\right)} & \frac{\beta_{1} S}{\gamma+\alpha_{4}} & \frac{\phi \beta_{1} S}{\left(\gamma+\alpha_{4}\right)\left(\gamma+\alpha_{3}+\phi\right)} \\
0 & 0 & 0 \\
0 & 0 & 0
\end{array}\right]
$$


Hence the basic reproductive number is deduced as

$$
R_{0}^{L}=\operatorname{trace}\left(D_{L}\right)=\frac{\epsilon \beta_{1} S\left[k\left(\gamma+\alpha_{3}+\phi\right)+\phi(1-k)\right]}{(\gamma+\epsilon+\xi)\left(\gamma+\alpha_{4}\right)\left(\gamma+\alpha_{3}+\phi\right)}
$$

\section{Case II: NGM with classical domain}

We let $\Psi=\left[\begin{array}{lll}1 & 0 & 0 \\ 0 & 0 & 0 \\ 0 & 0 & 0\end{array}\right]$, then $\Psi^{\prime} \Gamma=\left[\begin{array}{ccc}0 & \beta_{1} S & 0 \\ 0 & 0 & 0 \\ 0 & 0 & 0\end{array}\right],-\Delta^{-1} \Psi=\left[\begin{array}{ccc}\frac{1}{\gamma+\epsilon+\xi} & 0 & 0 \\ \frac{\epsilon k\left(\gamma+\alpha_{3}+\phi\right)+\phi \epsilon(1-k)}{(\gamma+\epsilon+\xi)\left(\gamma+\alpha_{3}+\phi\right)} & 0 & 0 \\ \frac{\epsilon(1-k)}{(\gamma+\epsilon+\xi)\left(\gamma+\alpha_{3}+\phi\right)} & 0 & 0\end{array}\right]$. The next-generation matrix is of the form $D_{C}=-\Psi^{\prime} \Gamma \Delta^{-1} \Psi=\left[\begin{array}{ccc}\frac{\epsilon \beta_{1} S\left[k\left(\gamma+\alpha_{3}+\phi\right)+\phi(1-k)\right]}{(\gamma+\epsilon+\xi)\left(\gamma+\alpha_{4}\right)\left(\gamma+\alpha_{3}+\phi\right)} & 0 & 0 \\ 0 & 0 & 0 \\ 0 & 0 & 0\end{array}\right]$. Then $R_{0}^{C}=\operatorname{trace}\left(D_{C}\right)=\frac{\epsilon \beta_{1} S\left[k\left(\gamma+\alpha_{3}+\phi\right)+\phi(1-k)\right]}{(\gamma+\epsilon+\xi)\left(\gamma+\alpha_{4}\right)\left(\gamma+\alpha_{3}+\phi\right)}$

\section{Case III: NGM with small domain}

The transmission matrix is decomposed as $\Omega=\left[\begin{array}{lll}0 & \beta_{1} & 0\end{array}\right]$ and $\Lambda=\left[\begin{array}{lll}S & 0 & 0\end{array}\right]^{\prime}$. Then

$$
-\Omega \Delta^{-1}=\left[\begin{array}{lll}
\frac{\epsilon \beta_{1}\left[k\left(\gamma+\alpha_{3}+\phi\right)+\phi(1-k)\right]}{(\gamma+\epsilon+\xi)\left(\gamma+\alpha_{4}\right)\left(\gamma+\alpha_{3}+\phi\right)} & \frac{\beta_{1}}{\gamma+\alpha_{4}} & \frac{\beta_{1} \theta}{\left(\gamma+\alpha_{4}\right)\left(\gamma+\alpha_{3}+\phi\right)}
\end{array}\right]
$$

and

$$
R_{0}^{S}=D_{S}=-\Omega \Delta^{-1} \Lambda=\frac{\epsilon \beta_{1} S\left[k\left(\gamma+\alpha_{3}+\phi\right)+\phi(1-k)\right]}{(\gamma+\epsilon+\xi)\left(\gamma+\alpha_{4}\right)\left(\gamma+\alpha_{3}+\phi\right)}
$$

\subsection{SIR Model with Quarantine}

The model equation for this Covid-19 characterization is given by

$$
\left\{\begin{array}{l}
S^{\prime}(t)=\mu N-\beta S I-\gamma S ; \\
I^{\prime}(t)=\beta S I-\left[\gamma+\theta_{1}+\alpha_{1}\right] I \\
Q^{\prime}(t)=\theta_{1} I-\left(\gamma+\alpha_{2}\right) Q ; \\
R^{\prime}(t)=\alpha_{1} I+\alpha_{2} Q-\gamma R .
\end{array}\right.
$$

For the infected states, then Jacobian matrix is $J(I, Q)=\left[\begin{array}{cc}\beta S-\left(\gamma+\theta_{1}+\alpha_{1}\right) & 0 \\ \theta_{1} & -\left(\gamma+\alpha_{2}\right)\end{array}\right]$. Then the transition and transmission matrices are deduced as $\Gamma=\left[\begin{array}{cc}\beta S & 0 \\ 0 & 0\end{array}\right], \Delta=\left[\begin{array}{cc}-\left(\gamma+\theta_{1}+\alpha_{1}\right) & 0 \\ \alpha_{1} & -\left(\gamma+\alpha_{2}\right.\end{array}\right]$, with the inverse matrix $\Delta^{-1}=\left[\begin{array}{cc}\frac{-1}{\gamma+\theta_{1}+\alpha_{1}} & 0 \\ \frac{-\theta_{1}}{\left(\gamma+\alpha_{2}\right)\left(\gamma+\alpha_{2}\right.} & \frac{-1}{\gamma+\alpha_{2}}\end{array}\right]$

\section{Case I: NGM with large domain}

$$
D_{L}=\left[\begin{array}{cc}
\frac{\beta S}{\gamma+\theta_{1}+\alpha_{1}} & 0 \\
0 & 0
\end{array}\right] \quad \text { and } \quad R_{0}^{L}=\frac{\beta S}{\gamma+\theta_{1}+\alpha_{1}}
$$




\section{Case II: NGM with classical domain}

We let $\Psi=\left[\begin{array}{ll}1 & 0 \\ 0 & 0\end{array}\right]$, then $\Psi^{\prime} \Gamma=\left[\begin{array}{cc}\beta S & 0 \\ 0 & 0\end{array}\right]$, and $-\Delta^{-1} \Psi=\left[\begin{array}{cc}\frac{1}{\gamma+\theta_{1}+\alpha_{1}} & 0 \\ \frac{\theta_{1}}{\left(\gamma+\alpha_{2}\right)\left(\gamma+\theta_{1}+\alpha_{1}\right)} & 0\end{array}\right]$.

The next-generation matrix for the classical domain and its corresponding basic reproductive number are respectively given by $D_{C}$ and $R_{0}^{C}$ below.

$$
D_{C}=\left[\begin{array}{cc}
\frac{\beta S}{\gamma+\theta_{1}+\alpha_{1}} & 0 \\
0 & 0
\end{array}\right] \text { and } R_{0}^{C}=\frac{\beta S}{\gamma+\theta_{1}+\alpha_{1}}
$$

\section{Case III: NGM with small domain}

The decomposed matrices are of the form $\Omega=\left[\begin{array}{ll}\beta & 0\end{array}\right], \Lambda=\left[\begin{array}{ll}S & 0\end{array}\right]^{\prime}$, then $-\Omega \Delta^{-1}=\left[\begin{array}{ll}\frac{\beta}{\gamma+\theta_{1}+\alpha_{1}} & 0\end{array}\right]$. Here, the basic reproductive number is equal to the spectral formula and is given by

$$
R_{0}^{S}=D_{S}=-\Omega \Delta^{-1} \Lambda=\frac{\beta S}{\gamma+\theta_{1}+\alpha_{1}} .
$$

\subsection{SEIR Model with Quarantine}

The model equations for the SEIR with quarantine is given by (11):

$$
\begin{cases}S^{\prime}(t) & =\mu N-\beta_{1} S I-\gamma S+\xi E \\ E^{\prime}(t) & \left.=\beta_{1} S I-\left[\gamma+\theta_{2}+\epsilon+\xi\right)\right] E \\ I^{\prime}(t) & =\epsilon E-\left[\gamma+\theta_{1}+\alpha_{1}\right] I \\ Q^{\prime}(t) & =\theta_{1} I+\theta_{2} E-\left(\gamma+\alpha_{2}\right) Q \\ R^{\prime}(t) & =\alpha_{1} I+\alpha_{2} Q-\gamma R\end{cases}
$$

The Jacobian matrix for the infected sub-population is

$$
J(E, I, Q)=\left[\begin{array}{ccc}
-\left(\gamma+\theta_{2}+\epsilon+\xi\right) & \beta_{1} S & 0 \\
\epsilon & -\left(\gamma+\theta_{1}+\alpha_{1}\right) & 0 \\
\theta_{2} & \theta_{1} & -\left(\gamma+\alpha_{2}\right)
\end{array}\right]
$$

The above matrix is decomposed as

$$
\Gamma=\left[\begin{array}{ccc}
0 & \beta_{1} S & 0 \\
0 & 0 & 0 \\
0 & 0 & 0
\end{array}\right], \Delta=\left[\begin{array}{ccc}
-\left(\gamma+\theta_{2}+\epsilon+\xi\right) & 0 & 0 \\
\epsilon & -\left(\gamma+\theta_{1}+\alpha_{1}\right) & 0 \\
\theta_{2} & \theta_{1} & -\left(\gamma+\alpha_{2}\right)
\end{array}\right]
$$

This yields the inverse of the transition matrix as

$$
\Delta^{-1}=\left[\begin{array}{ccc}
\frac{-1}{\gamma+\theta_{2}+\epsilon+\xi} & 0 & 0 \\
\left.\frac{\epsilon}{\left(\gamma+\theta_{2}+\epsilon+\xi\right)\left(\gamma+\theta_{1}+\alpha_{1}\right.}\right) & \frac{-1}{\gamma+\theta_{1}+\alpha_{1}} & 0 \\
\frac{\epsilon \theta_{1}+\theta_{2}\left(\gamma+\theta_{1}+\alpha_{1}\right)}{-\left(\gamma+\theta_{2}+\epsilon+\xi\right)\left(\gamma+\theta_{1}+\alpha_{1}\right)\left(\gamma+\alpha_{2}\right)} & \frac{-\theta_{1}}{\left(\gamma+\theta_{1}+\alpha_{1}\right)\left(\gamma+\alpha_{2}\right)} & \frac{-1}{\left(\gamma+\alpha_{2}\right)}
\end{array}\right] .
$$

\section{Case I: NGM with large domain}

For this case, the NGM; $D_{L}=\left[\begin{array}{ccc}\frac{\epsilon \beta_{1} S}{\left(\gamma+\theta_{2}+\epsilon+\xi\right)\left(\gamma+\theta_{1}+\alpha_{1}\right)} & \frac{\beta_{1} S}{\gamma+\theta_{1}+\alpha_{1}} & 0 \\ 0 & 0 & 0 \\ 0 & 0 & 0\end{array}\right]$ and $R_{0}^{L}=$ $\frac{\epsilon \beta_{1} S}{\left(\gamma+\theta_{2}+\epsilon+\xi\right)\left(\gamma+\theta_{1}+\alpha_{1}\right)}$. 


\section{Case II: NGM with classical domain}

Letting $\Psi=\left[\begin{array}{lll}1 & 0 & 0 \\ 0 & 0 & 0 \\ 0 & 0 & 0\end{array}\right]$, then $D_{C}=-\Psi^{\prime} \Gamma \Delta^{-1} \Psi=\left[\begin{array}{ccc}\frac{\epsilon \beta_{1} S}{\left(\gamma+\theta_{2}+\epsilon+\xi\right)\left(\gamma+\theta_{1}+\alpha_{1}\right)} & 0 & 0 \\ 0 & 0 & 0 \\ 0 & 0 & 0\end{array}\right]$. Thus, the basic reproductive number for this case is $R_{0}^{C}=\frac{\epsilon \beta_{1} S}{\left(\gamma+\theta_{2}+\epsilon+\xi\right)\left(\gamma+\theta_{1}+\alpha_{1}\right)}$.

\section{Case III: NGM with classical domain}

The matrix $\Gamma$ is break-down as $\Omega=\left[\begin{array}{lll}0 & \beta_{1} & 0\end{array}\right]$, and $\Lambda=\left[\begin{array}{ccc}S & 0 & 0\end{array}\right]^{\prime}$. Then

$$
-\Omega \Delta^{-1}=\left[\begin{array}{lll}
\frac{\epsilon \beta_{1}}{\left(\gamma+\theta_{2}+\epsilon+\xi\right)\left(\gamma+\theta_{1}+\alpha_{1}\right)} & \frac{\beta_{1}}{\left(\gamma+\theta_{1}+\alpha_{1}\right)} & \frac{\beta_{1} \theta_{1}}{\left(\gamma+\theta_{1}+\alpha_{1}\right)\left(\gamma+\alpha_{2}\right)}
\end{array}\right]
$$

and

$$
R_{0}^{S}=D_{S}=\frac{\epsilon \beta_{1} S}{\left(\gamma+\theta_{2}+\epsilon+\xi\right)\left(\gamma+\theta_{1}+\alpha_{1}\right)} .
$$

In subsequent derivations, the mathematics of the next-generation matrix are evinced without enough emphasis to the use of words. Besides, the $R_{0}$ results are obtained for all three domain cases.

\subsection{SIR Model with Quarantine and Lockdown}

This corona characterization is represented with the differential Equation (12).

$$
\left\{\begin{array}{l}
S^{\prime}(t)=\mu N-\beta S I-(\gamma+\eta) S+\delta L \\
L^{\prime}(t)=\eta S-(\gamma+\delta) L \\
I^{\prime}(t)=\beta S I-\left[\gamma+\theta_{1}+\alpha_{1}\right] I \\
Q^{\prime}(t)=\theta_{1} I-\left(\gamma+\alpha_{2}\right) Q \\
R^{\prime}(t)=\alpha_{1} I+\alpha_{2} Q-\gamma R .
\end{array}\right.
$$

Considering only the infected states, the matrix of first partial derivative is given by

$$
J(I, Q)=\left[\begin{array}{cc}
\beta S-\left(\gamma+\theta_{1}+\alpha_{1}\right) & 0 \\
\theta_{1} & -\left(\gamma+\alpha_{2}\right)
\end{array}\right]
$$

From the Jacobian matrix, the required components $\Delta$, and $\Gamma$ are obtained as $\Gamma=\left[\begin{array}{cc}\beta S & 0 \\ 0 & 0\end{array}\right]$, and $\Delta=$ $\left[\begin{array}{cc}-\left(\gamma+\theta_{1}+\alpha_{1}\right) & 0 \\ \alpha_{1} & -\left(\gamma+\alpha_{2}\right.\end{array}\right]$, with the inverse matrix $\Delta^{-1}=\left[\begin{array}{cc}\frac{-1}{\gamma+\theta_{1}+\alpha_{1}} & 0 \\ \frac{-\theta_{1}}{\left(\gamma+\alpha_{2}\right)\left(\gamma+\alpha_{2}\right.} & \frac{-1}{\gamma+\alpha_{2}}\end{array}\right]$.

\section{Case I: NGM with large domain}

$$
D_{L}=\left[\begin{array}{cc}
\frac{\beta S}{\gamma+\theta_{1}+\alpha_{1}} & 0 \\
0 & 0
\end{array}\right] \quad \text { and } \quad R_{0}^{L}=\frac{\beta S}{\gamma+\theta_{1}+\alpha_{1}}
$$

\section{Case II: NGM with classical domain}

$$
D_{C}=\left[\begin{array}{cc}
\frac{\beta S}{\gamma+\theta_{1}+\alpha_{1}} & 0 \\
0 & 0
\end{array}\right] \quad \text { and } \quad R_{0}^{C}=\frac{\beta S}{\gamma+\theta_{1}+\alpha_{1}}
$$

where $\Psi=\left[\begin{array}{ll}1 & 0 \\ 0 & 0\end{array}\right]$ 


\section{Case III: NGM with small domain}

$$
R_{0}^{S}=D_{S}=-\Omega \Delta^{-1} \Lambda=\frac{\beta S}{\gamma+\theta_{1}+\alpha_{1}}
$$

where $\Omega=\left[\begin{array}{ll}\beta & 0\end{array}\right]$, and $\Lambda=\left[\begin{array}{ll}S & 0\end{array}\right]^{\prime}$.

\subsection{SEIR Model with Quarantine and Lockdown}

The model equations are

$$
\begin{cases}S^{\prime}(t) & =\mu N-\beta_{1} S I-(\gamma+\eta) S+\delta L+\xi E \\ L^{\prime}(t) & =\eta S-(\gamma+\delta) L \\ E^{\prime}(t) & =\beta_{1} S I-\left[\gamma+\theta_{2}+\epsilon+\xi\right] E \\ I^{\prime}(t) & =\epsilon E-\left[\gamma+\theta_{1}+\alpha_{1}\right] I \\ Q^{\prime}(t) & =\theta_{1} I+\theta_{2} E-\left(\gamma+\alpha_{2}\right) Q \\ R^{\prime}(t) & =\alpha_{1} I+\alpha_{2} Q-\gamma R .\end{cases}
$$

The Jacobian for the infected states is

$$
J(E, I, Q)=\left[\begin{array}{ccc}
-\left(\gamma+\theta_{2}+\epsilon+\xi\right) & \beta_{1} S & 0 \\
\epsilon & -\left(\gamma+\theta_{1}+\alpha_{1}\right) & 0 \\
\theta_{2} & \theta_{1} & -\left(\gamma+\alpha_{2}\right)
\end{array}\right]
$$

Then $\Gamma=\left[\begin{array}{ccc}0 & \beta_{1} S & 0 \\ 0 & 0 & 0 \\ 0 & 0 & 0\end{array}\right], \Delta=\left[\begin{array}{ccc}-\left(\gamma+\theta_{2}+\epsilon+\xi\right) & 0 & 0 \\ \epsilon & -\left(\gamma+\theta_{1}+\alpha_{1}\right) & 0 \\ \theta_{2} & \theta_{1} & -\left(\gamma+\alpha_{2}\right)\end{array}\right]$, and $\Delta^{-1}=$ $\left[\begin{array}{ccc}\frac{-1}{\gamma+\theta_{2}+\epsilon+\xi} & 0 & 0 \\ \left.\frac{\epsilon}{\left(\gamma+\theta_{2}+\epsilon+\xi\right)\left(\gamma+\theta_{1}+\alpha_{1}\right.}\right) & \frac{-1}{\gamma+\theta_{1}+\alpha_{1}} & 0 \\ \frac{\epsilon \theta_{1}+\theta_{2}\left(\gamma+\theta_{1}+\alpha_{1}\right)}{-\left(\gamma+\theta_{2}+\epsilon+\xi\right)\left(\gamma+\theta_{1}+\alpha_{1}\right)\left(\gamma+\alpha_{2}\right)} & \frac{-\theta_{1}}{\left(\gamma+\theta_{1}+\alpha_{1}\right)\left(\gamma+\alpha_{2}\right)} & \frac{-1}{\left(\gamma+\alpha_{2}\right)}\end{array}\right]$

\section{Case I: NGM with large domain}

$$
D_{L}=\left[\begin{array}{ccc}
\frac{\epsilon \beta_{1} S}{\left(\gamma+\theta_{2}+\epsilon+\xi\right)\left(\gamma+\theta_{1}+\alpha_{1}\right)} & \frac{\beta_{1} S}{\gamma+\theta_{1}+\alpha_{1}} & 0 \\
0 & 0 & 0 \\
0 & 0 & 0
\end{array}\right], \quad R_{0}^{L}=\frac{\epsilon \beta_{1} S}{\left(\gamma+\theta_{2}+\epsilon+\xi\right)\left(\gamma+\theta_{1}+\alpha_{1}\right)}
$$

\section{Case II: NGM with classical domain}

$$
D_{C}=\left[\begin{array}{ccc}
\frac{\epsilon \beta_{1} S}{\left(\gamma+\theta_{2}+\epsilon+\xi\right)\left(\gamma+\theta_{1}+\alpha_{1}\right)} & 0 & 0 \\
0 & 0 & 0 \\
0 & 0 & 0
\end{array}\right], \quad R_{0}^{C}=\frac{\epsilon \beta_{1} S}{\left(\gamma+\theta_{2}+\epsilon+\xi\right)\left(\gamma+\theta_{1}+\alpha_{1}\right)}
$$

The entries of $\Psi$ are the same as the SEIR model with quarantine.

\section{Case III: NGM with small domain}

$$
R_{0}^{S}=D_{S}=-\Omega \Delta^{-1} \Lambda=\frac{\epsilon \beta_{1} S}{\left(\gamma+\theta_{2}+\epsilon+\xi\right)\left(\gamma+\theta_{1}+\alpha_{1}\right)}
$$

The entries of $\Omega$ and $\Lambda$ are the same as the SEIR model with quarantine. 


\subsection{SIR Model with Quarantine and Vaccination}

The SIR equations with quarantine and vaccinations are expressed as (14).

$$
\left\{\begin{array}{l}
S^{\prime}(t)=\mu N-\beta S I-\left(\gamma+\omega_{1}\right) S \\
V^{\prime}(t)=\omega_{1} S-\gamma V \\
I^{\prime}(t)=\beta S I-\left[\gamma+\theta_{1}+\alpha_{1}\right] I \\
Q^{\prime}(t)=\theta_{1} I-\left(\gamma+\alpha_{2}\right) Q \\
R^{\prime}(t)=\alpha_{1} I-\gamma R+\alpha_{2} Q .
\end{array}\right.
$$

For the infected sub-compartments, the Jacobian is mathematically expressed as

$$
J(I, Q)=\left[\begin{array}{cc}
\beta S-\left(\gamma+\theta_{1}+\alpha_{1}\right) & 0 \\
\theta_{1} & -\left(\gamma+\alpha_{2}\right)
\end{array}\right] .
$$

The transition, transmission and inverse matrices are

$$
\Gamma=\left[\begin{array}{cc}
\beta S & 0 \\
0 & 0
\end{array}\right], \Delta=\left[\begin{array}{cc}
-\left(\gamma+\theta_{1}+\alpha_{1}\right) & 0 \\
\alpha_{1} & -\left(\gamma+\alpha_{2}\right.
\end{array}\right], \Delta^{-1}=\left[\begin{array}{cc}
\frac{-1}{\gamma+\theta_{1}+\alpha_{1}} & 0 \\
\frac{-\theta_{1}}{\left(\gamma+\alpha_{2}\right)\left(\gamma+\alpha_{2}\right.} & \frac{-1}{\gamma+\alpha_{2}}
\end{array}\right]
$$

\section{Case I: NGM with large domain}

$$
D_{L}=\left[\begin{array}{cc}
\frac{\beta S}{\gamma+\theta_{1}+\alpha_{1}} & 0 \\
0 & 0
\end{array}\right] \quad \text { and } \quad R_{0}^{L}=\frac{\beta S}{\gamma+\theta_{1}+\alpha_{1}}
$$

\section{Case II: NGM with classical domain}

$$
D_{C}=\left[\begin{array}{cc}
\frac{\beta S}{\gamma+\theta_{1}+\alpha_{1}} & 0 \\
0 & 0
\end{array}\right] \text { and } R_{0}^{C}=\frac{\beta S}{\gamma+\theta_{1}+\alpha_{1}} .
$$

The entries of $\Psi$ are the same as the SIR model with quarantine.

\section{Case III: NGM with small domain}

$$
R_{0}^{S}=D_{S}=-\Omega \Delta^{-1} \Lambda=\frac{\beta S}{\gamma+\theta_{1}+\alpha_{1}} .
$$

The entries of $\Omega$ and $\Lambda$ are the same as the SEIR model with quarantine.

\subsection{SEIR Model with Quarantine and Vaccination}

The SEIR model with quarantine and vaccinations is given by the system (15).

$$
\left\{\begin{array}{l}
S^{\prime}(t)=\mu N-\beta_{1} S I-\left(\gamma+\omega_{1}\right) S+\xi E \\
V^{\prime}(t)=\omega_{1} S-\gamma V \\
E^{\prime}(t)=\beta_{1} S I-\left[\gamma+\theta_{2}+\epsilon+\xi\right] E \\
I^{\prime}(t)=\epsilon E-\left[\gamma+\theta_{1}-\alpha_{1}\right] I \\
Q^{\prime}(t)=\theta_{1} I+\theta_{2} E-\left(\gamma+\alpha_{2}\right) Q \\
R^{\prime}(t)=\alpha_{1} I-\gamma R+\alpha_{2} Q
\end{array}\right.
$$

For the infected states

$$
J(E, I, Q)=\left[\begin{array}{ccc}
-\left(\gamma+\theta_{2}+\epsilon+\xi\right) & \beta_{1} S & 0 \\
\epsilon & -\left(\gamma+\theta_{1}+\alpha_{1}\right) & 0 \\
\theta_{2} & \theta_{1} & -\left(\gamma+\alpha_{2}\right)
\end{array}\right]
$$


and

$$
\begin{gathered}
\Gamma=\left[\begin{array}{ccc}
0 & \beta_{1} S & 0 \\
0 & 0 & 0 \\
0 & 0 & 0
\end{array}\right], \Delta=\left[\begin{array}{ccc}
-\left(\gamma+\theta_{2}+\epsilon+\xi\right) & 0 & 0 \\
\epsilon & -\left(\gamma+\theta_{1}+\alpha_{1}\right) & 0 \\
\theta_{2} & \theta_{1} & -\left(\gamma+\alpha_{2}\right)
\end{array}\right] \\
\Delta^{-1}=\left[\begin{array}{ccc}
\frac{-1}{\gamma+\theta_{2}+\epsilon+\xi} & 0 & 0 \\
\left.\frac{\epsilon}{\left(\gamma+\theta_{2}+\epsilon+\xi\right)\left(\gamma+\theta_{1}+\alpha_{1}\right.}\right) & \frac{-1}{\gamma+\theta_{1}+\alpha_{1}} & 0 \\
\frac{\epsilon \theta_{1}+\theta_{2}\left(\gamma+\theta_{1}+\alpha_{1}\right)}{-\left(\gamma+\theta_{2}+\epsilon+\xi\right)\left(\gamma+\theta_{1}+\alpha_{1}\right)\left(\gamma+\alpha_{2}\right)} & \frac{-\theta_{1}}{\left(\gamma+\theta_{1}+\alpha_{1}\right)\left(\gamma+\alpha_{2}\right)} & \frac{-1}{\left(\gamma+\alpha_{2}\right)}
\end{array}\right] .
\end{gathered}
$$

\section{Case I: NGM with large domain}

$$
D_{L}=\left[\begin{array}{ccc}
\frac{\epsilon \beta_{1} S}{\left(\gamma+\theta_{2}+\epsilon+\xi\right)\left(\gamma+\theta_{1}+\alpha_{1}\right)} & \frac{\beta_{1} S}{\gamma+\theta_{1}+\alpha_{1}} & 0 \\
0 & 0 & 0 \\
0 & 0 & 0
\end{array}\right], \quad R_{0}^{L}=\frac{\epsilon \beta_{1} S}{\left(\gamma+\theta_{2}+\epsilon+\xi\right)\left(\gamma+\theta_{1}+\alpha_{1}\right)}
$$

\section{Case II: NGM with classical domain}

$$
D_{C}=\left[\begin{array}{ccc}
\frac{\epsilon \beta_{1} S}{\left(\gamma+\theta_{2}+\epsilon+\xi\right)\left(\gamma+\theta_{1}+\alpha_{1}\right)} & 0 & 0 \\
0 & 0 & 0 \\
0 & 0 & 0
\end{array}\right], \quad R_{0}^{C}=\frac{\epsilon \beta_{1} S}{\left(\gamma+\theta_{2}+\epsilon+\xi\right)\left(\gamma+\theta_{1}+\alpha_{1}\right)} .
$$

\section{Case III: NGM with small domain}

$$
R_{0}^{S}=D_{S}=\frac{\epsilon \beta_{1} S}{\left(\gamma+\theta_{2}+\epsilon+\xi\right)\left(\gamma+\theta_{1}+\alpha_{1}\right)} .
$$

These $R_{0}$ equations presented in this section explain the transmissibility of the coronavirus disease under several varied conditions. The choice will depend on the underlining characteristic of the coronavirus disease within the host population.

\section{Conclusion}

Since the outbreak of the coronavirus diseases, its endemicity is inferred from the $R_{0}$ of simple SIR and SEIR models. In some recent corona model offshoots; lockdowns, quarantine, and vaccination compartments have been introduced to reflect the current state of Covid-19.

In this paper, we derived the basic reproductive equations from these improved models to fully characterize the spread of the coronavirus disease within the informative stages. In section three, divergent $R_{0}$ formulas were presented depending on the state of the Covid-19 infections, and the measures implemented to curb the spread of the virus.

The next-generation matrix was used to derive these basic reproductive equations. In each case, the $R_{0}$ equation was derived using three different NGM domains; the classical domain, large domain, and small domain. These unique domain analyses yielded the same $R_{0}$ equations. This was corroborated with two propositional proofs.

With a better insight on the spread and control of the coronavirus disease within the informative phases of the corona disease, stakeholders could opt for a suitable $R_{0}$ equation among these to appropriately report on the transmissibility of SARS-CoV-2.

Author Contributions: All authors contributed equally in writing of this paper. All authors read and approved the final manuscript.

Conflicts of Interest: "The authors declare no conflict of interest."

\section{References}


[1] Fosu, G. O., Akweittey, E., \& Mensah, E. K. (2019). Modeling sexual activities among students; a global analysis. International Journal of Mathematics and Computation, 30(4).

[2] Diekmann, O., Heesterbeek, J. A. P., \& Roberts, M. G. (2010). The construction of next-generation matrices for compartmental epidemic models. Journal of the Royal Society Interface, 7(47), 873-885.

[3] Ma, J. (2020). Estimating epidemic exponential growth rate and basic reproduction number. Infectious Disease Modelling, 5, 129-141.

[4] Li, Z., Wu, M., Yao, J., Guo, J., Liao, X., Song, S., ... \& Zhou, Z. (2020). Caution on kidney dysfunctions of COVID-19 patients. medRxiv. 2020 2020.2002.2008.20021212.

[5] Zhao, S., Lin, Q., Ran, J., Musa, S. S., Yang, G., Wang, W., ... \& Wang, M. H. (2020). Preliminary estimation of the basic reproduction number of novel coronavirus (2019-nCoV) in China, from 2019 to 2020: A data-driven analysis in the early phase of the outbreak. International Journal of Infectious Diseases, 92, 214-217.

[6] Riou, J., \& Althaus, C. L. (2020). Pattern of early human-to-human transmission of Wuhan 2019 novel coronavirus (2019-nCoV), December 2019 to January 2020. Eurosurveillance, 25(4), 2000058.

[7] Wu, J. T., Leung, K., \& Leung, G. M. (2020). Nowcasting and forecasting the potential domestic and international spread of the 2019-nCoV outbreak originating in Wuhan, China: a modelling study. The Lancet, 395(10225), 689-697.

[8] Obeng-Denteh, W., Obeng, B. A., \& Fosu, G. O. (2015). On modelling of promiscuous lifestyle; An SIR dynamics. Journal of Advances in Mathematics and Computer Science, 475-480.

[9] Obeng, B. A., Fosu, G. O., \& Akweittey, E. (2016). A Mathematical Model on the Dynamics of Student-Lecturer-Sex on Campuses. Advances in Research, 6(5), 1-8.

[10] Obeng, B. A., Obeng-Denteh, W., Fosu, G. O., \& Yaro, D. (2014). An epidemiological model for sexual activities. International Journal of Sciences: Basic and Applied Research, 17(1), 210-214.

[11] D'Arienzo, M., \& Coniglio, A. (2020). Assessment of the SARS-CoV-2 basic reproduction number, R0, based on the early phase of COVID-19 outbreak in Italy. Biosafety and Health, 2(2), 57-59.

[12] Sahafizadeh, E., \& Sartoli, S. (2020). Estimating the reproduction number of COVID-19 in Iran using epidemic modeling. medrxiv.org/content/10.1101/2020.03.20.20038422v3.

[13] Zhou, T., Liu, Q., Yang, Z., Liao, J., Yang, K., Bai, W., ... \& Zhang, W. (2020). Preliminary prediction of the basic reproduction number of the Wuhan novel coronavirus 2019-nCoV. Journal of Evidence-Based Medicine, 13(1), 3-7.

[14] Fosu, G. O., Opong, J. M., \& Appati, J. K. (2020). Construction of Compartmental Models for COVID-19 with Quarantine, Lockdown and Vaccine Interventions. Lockdown and Vaccine Interventions (April 12, 2020). http://dx.doi.org/10.2139/ssrn.3574020.

[15] Diekmann, O., Heesterbeek, J. A. P., \& Metz, J. A. (1990). On the definition and the computation of the basic reproduction ratio $R_{0}$ in models for infectious diseases in heterogeneous populations. Journal of Mathematical Biology, 28(4), 365-382.

[16] Van den Driessche, P., \& Watmough, J. (2002). Reproduction numbers and sub-threshold endemic equilibria for compartmental models of disease transmission. Mathematical biosciences, 180(1-2), 29-48. 